\title{
Hilbert Space Representations of Generalized Canonical Commutation Relations
}

\author{
Asao Arai \\ Department of Mathematics, Hokkaido University, Sapporo 060-0810, Japan \\ Correspondence should be addressed to Asao Arai; arai@math.sci.hokudai.ac.jp
}

Received 19 November 2012; Accepted 10 December 2012

Academic Editor: Stefan Siegmund

Copyright (C) 2013 Asao Arai. This is an open access article distributed under the Creative Commons Attribution License, which permits unrestricted use, distribution, and reproduction in any medium, provided the original work is properly cited.

We consider Hilbert space representations of a generalization of canonical commutation relations (CCRs) : $\left[X_{j}, X_{k}\right]:=X_{j} X_{k}-$ $X_{k} X_{j}=i \Theta_{j k} I(j, k=1,2, \ldots, 2 n)$, where $X_{j}$ 's are the elements of an algebra with identity $I, i$ is the imaginary unit, and $\Theta_{j k}$ is a real number with antisymmetry $\Theta_{j k}=-\Theta_{k j}(k, j=1,2, \ldots, 2 n)$. Some basic aspects on Hilbert space representations of the generalized CCR (GCCR) are discussed. We define a Schrödinger-type representation of the GCCR by an analogy with the usual Schrödinger representation of the CCR with $n$ degrees of freedom. Also, we introduce a Weyl-type representation of the GCCR. The main result of the present paper is a uniqueness theorem on Weyl representations of the GCCR.

\section{Introduction}

In this paper, we consider Hilbert space representations of a generalized canonical commutation relations (GCCRs) with $n$ degrees of freedom $(n \in \mathbb{N}:=\{1,2,3, \ldots\})$ of the following type:

$$
\left[X_{j}, X_{k}\right]=i \Theta_{j k} I \quad(j, k=1, \ldots, 2 n),
$$

where $X_{j}$ 's are elements of an algebra with identity $I$, $\left[X_{j}, X_{k}\right]:=X_{j} X_{k}-X_{k} X_{j}, i$ is the imaginary unit, and $\Theta_{j k} \in \mathbb{R}$ (the set of real numbers) with antisymmetry $\Theta_{j k}=$ $-\Theta_{k j}(j, k=1, \ldots, 2 n)$ such that, for some pair $(j, k), \Theta_{j k} \neq 0$. For convenience, we call (1) the $\Theta$-GCCR with $n$ degrees of freedom and the $2 n \times 2 n$ matrix

$$
\Theta:=\left(\Theta_{j k}\right)_{j, k=1, \ldots, 2 n}
$$

the noncommutative factor for $\left\{X_{j}\right\}_{j=1}^{2 n}$.

Note that, in the case where $\Theta$ is equal to

$$
J:=\left(\begin{array}{cc}
0 & I_{n} \\
-I_{n} & 0
\end{array}\right)
$$

with $I_{n}$ being the $n \times n$ unit matrix, (1) becomes the CCR with $n$ degrees of freedom. Namely, if we put $Q_{j}:=X_{j}, P_{j}:=$ $X_{n+j}(j=1, \ldots, n)$ in the present case, then we have

$$
\begin{aligned}
& {\left[Q_{j}, Q_{k}\right]=0, \quad\left[P_{j}, P_{k}\right]=0,} \\
& {\left[Q_{j}, P_{k}\right]=i \delta_{j k} I \quad(j, k=1, \ldots, n),}
\end{aligned}
$$

where $\delta_{j k}$ is the Kronecker delta. Thus, (1) is a natural generalization of the CCR with $n$ degrees of freedom.

The GCCR also includes some of non-commutative space times (e.g., [1-3]), non-commutative spaces (e.g., [4]), and non-commutative phase spaces (e.g., [5-11]). In fact, one of the motivations for the present work is to investigate general structures underlying those non-commutative objects. In this paper, however, we present only some fundamental aspects of Hilbert space representations of the GCCR. The main result is to establish a uniqueness theorem on Weyl type representations of the GCCR (for the definition, see Section $4)$.

In Section 2, we define Hilbert space representations of the GCCR and discuss some basic facts on them. It is shown that there exists a one-to-one correspondence between representations of the GCCR and the CCR with the same degrees of freedom. In Section 3, we introduce a Schrödingertype representation of the GCCR, whose representation space 
is $L^{2}\left(\mathbb{R}^{n}\right)$ as in the case of the Schrödinger representation of the CCR with $n$ degrees of freedom. In Section 4, Weyltype representations of the GCCR are defined by analogy with Weyl representations of CCR. In the last section, we prove the uniqueness theorem mentioned above. In Appendix, we present some basic properties of self-adjoint operators obeying generalized Weyl relations, which are used in the text.

\section{Basic Facts on Hilbert Space Representations of the $\Theta-$ GCCR}

Let $\mathscr{H}$ be a complex Hilbert space with inner product $\langle\cdot, \cdot\rangle$ (antilinear in the first variable and linear in the second one) and norm $\|\cdot\|$. For a linear operator $A$ on $\mathscr{H}$, we denote its domain by $D(A)$. For linear operators $A_{1}, \ldots, A_{p}$ on $\mathscr{H}$,

$$
\begin{aligned}
& D\left(\sum_{i=1}^{p} A_{i}\right):=\cap_{i=1}^{p} D\left(A_{i}\right), \\
& D\left(A_{1} A_{2}\right):=\left\{\Psi \in D\left(A_{2}\right) \mid A_{2} \Psi \in D\left(A_{1}\right)\right\}, \\
& D\left(A_{1} \cdots A_{p}\right):=D\left(\left(A_{1} \cdots A_{p-1}\right) A_{p}\right) \quad(p \geq 3) .
\end{aligned}
$$

Definition 1. Let $\mathscr{D}$ be a dense subspace of $\mathscr{H}$ and $X_{j}$, $j=1, \ldots, 2 n$, be symmetric (not necessarily essentially selfadjoint) operators on $\mathscr{H}$. Set $\mathbf{X}:=\left(X_{1}, \ldots, X_{2 n}\right)$. We say that the triple $(\mathscr{H}, \mathscr{D}, \mathbf{X})$ is a symmetric representation of the $\Theta$ GCCR with $n$ degrees of freedom if $\mathscr{D} \subset \cap_{j, k=1}^{2 n} D\left(X_{j} X_{k}\right)$ and (1) holds on $\mathscr{D}$.

If all the $X_{j}$ 's $(j=1, \ldots, 2 n)$ are self-adjoint, we say that $(\mathscr{H}, \mathscr{D}, \mathbf{X})$ is a self-adjoint representation of the GCCR.

Remark 2. The concept of self-adjoint representation defined above is different from the one used in representation theory of $*$-algebra (e.g., [12, page 205]).

Remark 3. In each symmetric representation $(\mathscr{H}, \mathscr{D}, \mathbf{X})$ of the $\Theta-\mathrm{GCCR}, \mathscr{H}$ is infinite dimensional (if $\mathscr{H}$ were finite dimensional, then, for $(j, k)$ such that $\Theta_{j k} \neq 0,0=$ trace of $\left[X_{j}, X_{k}\right]=i \Theta_{j k} \operatorname{dim} \mathscr{H} \neq 0$, and hence one is led to a contradiction).

Remark 4. It follows from a well-known fact on commutation properties of linear operators (e.g., [13, Theorem 1.2.3]) that, for $(j, k)$ with $\Theta_{j k} \neq 0$, at least one of $X_{j}$ and $X_{k}$ is unbounded. Hence, one has to be careful about domains of $X_{j}$ 's.

Remark 5. In the case of Hilbert space representations of CCR, symmetric representations, but nonself-adjoint ones, also play important roles. For example, such representations appear in mathematical theories of time operators [14] (see also $[15,16]$ for investigations from purely operator-theoretic points of view). Thus, it is expected that, in addition to self-adjoint representations of the $\Theta$-GCCR, non-self-adjoint symmetric representations of it may have any importance in applications to quantum physics.
Remark 6. In the context of quantum mechanics, for a symmetric operator $A$ and a unit vector $\psi \in D(A),(\Delta A)_{\psi}:=$ $\|(A-\langle\psi, A \psi\rangle) \psi\|$ is called the uncertainty of $A$ in the vector state $\psi$. Let $(\mathscr{H}, \mathscr{D}, \mathbf{X})$ be a symmetric representation of the $\Theta$-GCCR with $n$ degrees of freedom. Then, one has uncertainty relations of Robertson type [17]: for all unit vectors $\psi \in \mathscr{D}$ and $j, k=1, \ldots, 2 n$,

$$
\left(\Delta X_{j}\right)_{\psi}\left(\Delta X_{k}\right)_{\psi} \geq \frac{1}{2}\left|\left\langle\psi, \Theta_{j k} \psi\right\rangle\right| \text {. }
$$

Let $(\mathscr{H}, \mathscr{D}, \mathbf{X})$ be a symmetric representation of the $\Theta$ GCCR as in Definition 1. We assume for simplicity the following:

Assumption 1. The noncommutative factor $\Theta$ is regular (invertible).

Under this assumption, $\Theta$ is a regular antisymmetric real matrix. Hence, by a well-known fact in the theory of linear algebra (e.g., [18, page 173, Problem 9]), the following fact holds.

Lemma 7. There exists a regular $2 n \times 2 n$ real matrix $T_{0}$ such that ${ }^{t} T_{0} \Theta T_{0}=J$, where ${ }^{t} T_{0}$ is the transposed matrix of $T_{0}$ and $J$ is defined by (3).

The matrix $T_{0}$ in Lemma 7 belongs to the set

$$
\begin{aligned}
M_{\Theta}:= & \{T \mid T \text { is a } 2 n \times 2 n \\
& \text { real matrix such that } \left.{ }^{\mathrm{t}} T \Theta T=J\right\} .
\end{aligned}
$$

It is easy to see that for each $T \in M_{\Theta}$, there exists a unique $2 n \times 2 n$ symplectic matrix $W$ (i.e., ${ }^{\mathrm{t}} W J W=J$ ) such that $T=T_{0} W$. Hence,

$$
M_{\Theta}=\left\{\left.T_{0} W\right|^{\mathrm{t}} W J W=J\right\} .
$$

For a $2 n \times 2 n$ real matrix $L=\left(L_{j k}\right)_{j, k=1, \ldots, 2 n}$, we define

$$
X_{j}^{L}:=\sum_{k=1}^{2 n} L_{k j} X_{k}, \quad j=1, \ldots, 2 n .
$$

We call the correspondence $\mathbf{X} \mapsto \mathbf{X}^{L}:=\left(X_{1}^{L}, \ldots, X_{2 n}^{L}\right)$ the L-transform of $\mathbf{X}$.

Let

$$
\Theta_{L}:={ }^{\mathrm{t}} L \Theta L
$$

Proposition 8. (i) For all $j=1, \ldots, 2 n, X_{j}^{L}$ is a symmetric operator on $\mathscr{H}$.

(ii) For all $j, k=1, \ldots, 2 n$,

$$
\left[X_{j}^{L}, X_{k}^{L}\right]=i\left(\Theta_{L}\right)_{j k}
$$

on $\mathscr{D}$.

(iii) For each $T \in M_{\Theta}$ and $j, k=1, \ldots, 2 n$,

$$
\left[X_{j}^{T}, X_{k}^{T}\right]=i J_{j k}
$$

on $\mathscr{D}$. 
Proof. An easy exercise.

Proposition 8-(i) and (ii) show that $\left(\mathscr{H}, \mathscr{D}, \mathbf{X}^{L}\right)$ is a symmetric representation of the $\Theta_{L}$-GCCR with $n$ degrees of freedom.

Proposition 8 (iii) implies the following.

Corollary 9. Let $T \in M_{\Theta}$ and

$$
Q_{j}:=X_{j}^{T}, \quad P_{j}:=X_{n+j}^{T} \quad(j=1, \ldots, n) .
$$

Then, $\left(\mathscr{H}, \mathscr{D},\left\{Q_{j}, P_{j}\right\}_{j=1}^{n}\right)$ is a symmetric representation of the CCR with $n$ degrees of freedom.

Corollary 9 means that for each $T \in M_{\Theta}$, the $T$-transform of $\mathbf{X}$ gives a correspondence from a symmetric representation of the $\Theta$-GCCR with $n$ degrees of freedom to a symmetric representation of the CCR with the same degrees of freedom.

One can easily see that (9) with $L=T \in M_{\Theta}$ implies that

$$
X_{j}=\sum_{k=1}^{2 n}\left(T^{-1}\right)_{k j} X_{k}^{T}
$$

on $\cap_{j=1}^{2 n} D\left(X_{j}\right)$. Thus, every symmetric representation of the $\Theta-G C C R$ with $n$ degrees of freedom is constructed from a symmetric representation of the CCR with the same degrees of freedom via (14).

Conversely, if a symmetric representation $\left(\mathscr{H}, \mathscr{D},\left\{Q_{j}\right.\right.$, $\left.P_{j}\right\}_{j=1}^{n}$ ) of the CCR with $n$ degrees of freedom is given and let

$$
X_{j}(\mathbf{Q}, \mathbf{P} ; T):=\sum_{k=1}^{n}\left(T^{-1}\right)_{k j} Q_{k}+\sum_{k=1}^{n}\left(T^{-1}\right)_{(n+k) j} P_{k}
$$

with $\mathbf{Q}:=\left(Q_{1}, \ldots, Q_{n}\right)$ and $\mathbf{P}:=\left(P_{1}, \ldots, P_{n}\right)$, then $(\mathscr{H}, \mathscr{D}, \mathbf{X}(\mathbf{Q}, \mathbf{P} ; T))$ is a symmetric representation of the $\Theta$ GCCR and (9) holds with $L=T, X_{j}^{T}=Q_{j}, X_{n+j}^{T}=P_{j}(j=$ $1, \ldots, n)$ and $X_{j}=X_{j}(\mathbf{Q}, \mathbf{P} ; T)(j=1, \ldots, 2 n)$. Hence, every symmetric representation of the CCR with $n$ degrees of freedom is constructed from a symmetric representation of the $\Theta$-GCCR with the same degrees of freedom. Thus, for each $T \in M_{\Theta}$, there exists a one-to-one correspondence between a symmetric representation of the $\Theta-G C C R$ and a symmetric representation of the CCR with $n$ degrees of freedom.

\section{Representations of Schrödinger Type}

Let $T \in M_{\Theta}$. By the fact on $\mathbf{X}(\mathbf{Q}, \mathbf{P} ; T)$ stated in the preceding section, we can define a class of representations of the $\Theta$ GCCR. Let $\left(L^{2}\left(\mathbb{R}^{n}\right), C_{0}^{\infty}\left(\mathbb{R}^{n}\right),\left\{q_{j}, p_{j}\right\}_{j=1}^{n}\right)$ be the Schrödinger representation of the CCR with $n$ degrees of freedom, that is, $q_{j}$ is the multiplication operator by the $j$ th component $x_{j}$ of $\mathbf{x}=\left(x_{1}, \ldots, x_{n}\right) \in \mathbb{R}^{n}$ and $p_{j}:=-i D_{j}$ with $D_{j}$ being the generalized partial differential operator in $x_{j}$, acting in $L^{2}\left(\mathbb{R}^{n}\right)$. Let

$$
X_{j}(\mathbf{q}, \mathbf{p} ; T)=\sum_{k=1}^{n}\left(T^{-1}\right)_{k j} q_{k}+\sum_{k=1}^{n}\left(T^{-1}\right)_{(n+k) j} p_{k},
$$

which is (15) with $\mathbf{Q}=\mathbf{q}$ and $\mathbf{P}=\mathbf{p}$. We denote the closure of $X_{j}(\mathbf{q}, \mathbf{p} ; T)$ by $\bar{X}_{j}(\mathbf{q}, \mathbf{p} ; T)$ and set

$$
\overline{\mathbf{X}}(\mathbf{q}, \mathbf{p} ; T):=\left(\bar{X}_{1}(\mathbf{q}, \mathbf{p} ; T), \ldots, \bar{X}_{2 n}(\mathbf{q}, \mathbf{p} ; T)\right) .
$$

We call the triple $\pi_{S}^{T}:=\left(L^{2}\left(\mathbb{R}^{n}\right), C_{0}^{\infty}\left(\mathbb{R}^{n}\right), \overline{\mathbf{X}}(\mathbf{q}, \mathbf{p} ; T)\right)$ the $T$ Schrödinger representation of the $\Theta-G C C R$.

It is easy to see that for all $j=1, \ldots, 2 n, X_{j}(\mathbf{q}, \mathbf{p} ; T)$ is essentially self-adjoint on $C_{0}^{\infty}\left(\mathbb{R}^{n}\right)$ (apply, e.g., the Nelson commutator theorem [19, Theorem X.37] with dominating operator $N=\sum_{j=1}^{n}\left(q_{j}^{2}+p_{j}^{2}\right)+I$ ) (This can be proved also by applying Proposition 16$)$. Hence $\bar{X}_{j}(\mathbf{q}, \mathbf{p} ; T)$ is self-adjoint. Thus, we obtain the following.

Proposition 10. For each $T \in M_{\Theta}$, the $T$-Schrödinger representation $\pi_{S}^{T}$ is a self-adjoint representation of the $\Theta$ GCCR.

\section{Representations of Weyl Type}

Based on an analogy with Weyl representations of CCR, we introduce a concept of Weyl representation for $\Theta$-GCCR.

Definition 11. Let $\left\{X_{j}\right\}_{j=1}^{2 n}$ be a set of self-adjoint operators on a Hilbert space $\mathscr{H}$. We say that $\left\{X_{j}\right\}_{j=1}^{2 n}$ is a Weyl representation of the $\Theta-G C C R$ with $n$ degrees of freedom if for all $s, t \in \mathbb{R}$ and $j, k=1, \ldots, 2 n$,

$$
e^{i t X_{j}} e^{i s X_{k}}=e^{-i s t \Theta_{j k}} e^{i s X_{k}} e^{i t X_{j}} .
$$

We call these relations the $\Theta$-Weyl relations.

For a linear operator $A$ on a Hilbert space, we denote its spectrum by $\sigma(A)$.

Proposition 12. Let $\left\{X_{j}\right\}_{j=1}^{2 n}$ be a Weyl representation of the $\Theta-G C C R$ on $\mathscr{H}$. Then, there is a dense subspace $\mathscr{D}_{0} \subset \mathscr{H}$ left invariant by each $X_{j}(j=1, \ldots, 2 n)$ such that $\left(\mathscr{H}, \mathscr{D}_{0}, \mathbf{X}\right)$ is a self-adjoint representation of the $\Theta-G C C R$. Moreover, for every pair $\left(X_{j}, X_{k}\right)$ such that $\Theta_{j k} \neq 0, X_{j}$ and $X_{k}$ are purely absolutely continuous with

$$
\sigma\left(X_{j}\right)=\sigma\left(X_{k}\right)=\mathbb{R}, \quad j=1, \ldots, 2 n .
$$

Proof. By (18), we can apply the results described in the Appendix of the present paper. In the present context, we need only to take, in the notation in the Appendix, $N=$ $2 n, a_{j k}=\Theta_{j k}$ and $A_{j}=X_{j}$. By Proposition A.4-(iii) and Corollary A.5, there exists a dense subspace $\mathscr{D}_{0}$ left invariant by $X_{j}(j=1, \ldots, 2 n)$ and $\left[X_{j}, X_{k}\right]=i \Theta_{j k}$ on $\mathscr{D}_{0}$. Thus, the first half of the proposition is derived. The second half follows from Proposition A.1.

Remark 13. As in the case of self-adjoint representations of CCR (e.g., $[16,20,21])$, the converse of Proposition 12 does not hold (i.e., a self-adjoint representation of the $\Theta$-GCCR is not necessarily a Weyl one). 
We recall that a set $\left\{Q_{j}, P_{j}\right\}_{j=1}^{n}$ of self-adjoint operators on $\mathscr{H}$ is a Weyl representation of the CCR with $n$ degrees of freedom if for all $s, t \in \mathbb{R}$ and $j, k=1, \ldots, n$, the following Wey relations hold:

$$
\begin{aligned}
& e^{i t Q_{j}} e^{i s P_{k}}=e^{-i s t \delta_{j k}} e^{i s P_{k}} e^{i t Q_{j}}, \\
& e^{i t Q_{j}} e^{i s Q_{k}}=e^{i s Q_{k}} e^{i t Q_{j}}, \\
& e^{i t P_{j}} e^{i s P_{k}}=e^{i s P_{k}} e^{i t P_{j}} .
\end{aligned}
$$

Remark 14. A set $\left\{Q_{j}, P_{j}\right\}_{j=1}^{n}$ of self-adjoint operators on $\mathscr{H}$ is a Weyl representation of the CCR with $n$ degrees of freedom if and only if $\left\{X_{j}\right\}_{j=1}^{2 n}$ with $X_{j}:=Q_{j}, X_{n+j}=P_{j}(j=1, \ldots, n)$ is a Weyl representation of the $J$-GCCR, where $J$ is given by (3).

Let $T \in M_{\Theta}$ be arbitrarily fixed. The next proposition shows that the $T$-transform of each Weyl representation of the $\Theta-$ GCCR is a Weyl representation of the CCR with $n$ degrees of freedom.

Proposition 15. Let $\left\{X_{j}\right\}_{j=1}^{2 n}$ be a Weyl representation of the $\Theta-G C C R$ on $\mathscr{H}$, and let $\mathbf{X}^{T}$ be the T-transform of $\mathbf{X}$. Then, each $X_{j}^{T}$ is essentially self-adjoint, and $\left\{\overline{X_{j}^{T}}\right\}_{j=1}^{2 n}$ is a Weyl representation of the $J-G C C R$.

Proof. The essential self-adjointness of $X_{j}^{T}$ follows from a simple application of Theorem A.6 in Appendix. Corollary A.7 in Appendix and the relation ${ }^{\mathrm{t}} T \Theta T=J$ imply that $\left\{\overline{X_{j}^{T}}\right\}_{j=1}^{2 n}$ satisfies the $J$-Weyl relations.

In the same way as in the proof of Proposition 15, we can prove the following proposition:

Proposition 16. Let $\left\{Q_{j}, P_{j}\right\}_{j=1}^{n}$ be a Weyl representation of the CCR with $n$ degrees of freedom on a Hilbert space $\mathscr{H}$. Let $X_{j}(\mathbf{Q}, \mathbf{P} ; T)(j=1, \ldots, 2 n)$ be defined by (15). Then, each $X_{j}(\mathbf{Q}, \mathbf{P} ; T)$ is essentially self-adjoint and $\left\{\bar{X}_{j}(\mathbf{Q}, \mathbf{P} ; T)\right\}_{j=1}^{2 n}$ is a Weyl representation of $\Theta-G C C R$ with $n$ degrees of freedom.

This proposition shows that the converse of Proposition 15 holds too. Thus, for each $T \in M_{\Theta}$, there exists a one-to-one correspondence between a Weyl representation of the CCR with $n$ degrees of freedom and that of the $\Theta-$ GCCR with the same degrees of freedom.

It is well known [22] that the Schrödinger representation $\left\{q_{j}, p_{j}\right\}_{j=1}^{n}$ is a Weyl representation o the CCR with $n$ degrees of freedom. Hence, we obtain the following result.

Corollary 17. For each $T \in M_{\Theta}$, the $T$-Schrödinger representation $\left\{\bar{X}_{j}(\mathbf{q}, \mathbf{p} ; T)\right\}_{j=1}^{2 n}$ is a Weyl representation of the $\Theta$ GCCR.

We say that a Weyl representation $\left\{X_{j}\right\}_{j=1}^{2 n}$ of the $\Theta$-GCCR on $\mathscr{H}$ is irreducible if every closed subspace $\mathscr{M}$ of $\mathscr{H}$ which is invariant under the action of $e^{i t X_{j}}(t \in \mathbb{R}, j=1, \ldots, 2 n)$ is $\{0\}$ or $\mathscr{H}$.
Proposition 18. Let $T \in M_{\Theta}$. Then, the $T$-Schrödinger representation $\left\{\bar{X}_{j}(\mathbf{q}, \mathbf{p} ; T)\right\}_{j=1}^{2 n}$ as a Weyl representation of the $\Theta-G C C R$ is irreducible.

Proof. Let $\mathscr{M}$ be an invariant closed subspace of $e^{i t \bar{X}_{j}(q, p ; T)}$ $(t \in \mathbb{R}, j=1, \ldots, 2 n)$. We have

$$
\begin{aligned}
& q_{j}=\sum_{k=1}^{2 n} T_{k j} X_{k}(\mathbf{q}, \mathbf{p} ; T), \\
& p_{j}=\sum_{k=1}^{2 n} T_{k(n+j)} X_{k}(\mathbf{q}, \mathbf{p} ; T)
\end{aligned}
$$

on $\cap_{j=1}^{2 n} D\left(X_{j}(\mathbf{q}, \mathbf{p} ; T)\right)=\cap_{j=1}^{n} D\left(q_{j}\right) \cap D\left(p_{j}\right)$. Hence, by an application of Theorem A.6 in Appendix, $e^{i t q_{j}}$ and $e^{i t p_{j}}$ $(t \in \mathbb{R})$ can be written, respectively, as a scalar multiple of $e^{i t \bar{X}_{1}(\mathbf{q}, \mathbf{p} ; T)} \ldots e^{i t \bar{X}_{2 n}(\mathbf{q}, \mathbf{p} ; T)}$. Hence, $\mathscr{M}$ is invariant under the action of $e^{i t q_{j}}$ and $e^{i t p_{j}}(t \in \mathbb{R}, j=1, \ldots, n)$. It is well known that $\left\{e^{i t q_{j}}, e^{i t p_{j}} \mid t \in \mathbb{R}, j=1, \ldots, n\right\}$ is irreducible. Thus, $\mathscr{M}=\{0\}$ or $\mathscr{H}$.

\section{Uniqueness Theorem on Weyl Representations of the $\Theta-$ GCCR}

In this section, we prove the main result of the present paper, that is, a uniqueness theorem on Weyl representations of the $\Theta-$ GCCR, which may be regarded as a GCCR version of the celebrated von Neumann uniqueness theorem of Weyl representations of CCR ([13, Theorem 4.11.1], [22], [23, Theorem VIII.14]).

Theorem 19. Let $\left\{X_{j}\right\}_{j=1}^{2 n}$ be a Weyl representation of the $\Theta$ GCCR on a separable Hilbert space $\mathscr{H}$. Then, for each $T \in$ $M_{\Theta}$, there exist mutually orthogonal closed subspaces $\mathscr{H}_{\ell}(\ell=$ $1, \ldots, N ; N \in \mathbb{N}$ or $\infty$ ) such that the following (i)-(iii) hold.

(i) $\mathscr{H}=\oplus_{\ell=1}^{N} \mathscr{H}_{\ell}$.

(ii) For each $j=1, \ldots, 2 n, X_{j}$ is reduced by each $\mathscr{H}_{\ell}, \ell=$ $1, \ldots, N$. We denote by $X_{j}^{(\ell)}$ the reduced part of $X_{j}$ to $\mathscr{H}_{\ell}$.

(iii) For each $\ell$, there exists a unitary operator $U_{\ell}: \mathscr{H}_{\ell} \rightarrow$ $L^{2}\left(\mathbb{R}^{n}\right)$ such that

$$
U_{\ell} X_{j}^{(\ell)} U_{\ell}^{-1}=\bar{X}_{j}(\mathbf{q}, \mathbf{p} ; T), \quad j=1, \ldots, 2 n,
$$

where $\left\{\bar{X}_{j}(\mathbf{q}, \mathbf{p} ; T)\right\}_{j=1}^{2 n}$ is the T-Schrödinger representation of the $\Theta-G C C R$.

Proof. Let $T \in M_{\Theta}, \mathbf{X}^{T}$ be the $T$-transform of $\mathbf{X}$ and $Q_{j}:=$ $\overline{X_{j}^{T}}, P_{j}:=\overline{X_{n+j}^{T}}(j=1, \ldots, n)$. Then, by Proposition 15 and Remark 14, $\left\{Q_{j}, P_{j}\right\}_{j=1}^{n}$ is a Weyl representation of the CCR with $n$ degrees of freedom. Hence, by the von Neumann uniqueness theorem mentioned above, there exist mutually orthogonal closed subspaces $\mathscr{H}_{\ell}$ such that (i) given above and the following (a) and (b) hold. 
(a) For each $j=1, \ldots, n$ and all $t \in \mathbb{R}, e^{i t Q_{j}}$ and $e^{i t P_{j}}$ leave each $\mathscr{H}_{\ell}$ invariant $(\ell=1, \ldots, N)$.

(b) For each $\ell$, there exists a unitary operator $U_{\ell}: \mathscr{H}_{\ell} \rightarrow$ $L^{2}\left(\mathbb{R}^{n}\right)$ such that

$$
\begin{aligned}
& U_{\ell} e^{i t Q_{j}} U_{\ell}^{-1}=e^{i t q_{j}}, \\
& U_{\ell} e^{i t P_{j}} U_{\ell}^{-1}=e^{i t p_{j}}, \quad t \in \mathbb{R}, j=1, \ldots, n .
\end{aligned}
$$

By (14), we have $X_{j}=X_{j}(\mathbf{Q}, \mathbf{P} ; T)$ on $\cap_{j=1}^{2 n} D\left(X_{j}\right)$. Hence, $X_{j} \subset \bar{X}_{j}(\mathbf{Q}, \mathbf{P} ; T)$. By Proposition $16, \bar{X}_{j}(\mathbf{Q}, \mathbf{P} ; T)$ is selfadjoint. Hence, $X_{j}=\bar{X}_{j}(\mathbf{Q}, \mathbf{P} ; T)$. Therefore, by Theorem A.6 in Appendix, we obtain

$$
\begin{array}{r}
e^{i t X_{j}}=e^{i t^{2} \sum_{k<m}^{2 n} J_{k m}\left(T^{-1}\right)_{k j}\left(T^{-1}\right)_{m j} / 2} e^{i t Q_{1}} \cdots e^{i t Q_{n}} e^{i t P_{1}} \cdots e^{i t P_{n}}, \\
j=1, \ldots, 2 n .
\end{array}
$$

Hence, each $e^{i t X_{j}}$ leaves $\mathscr{H}_{\ell}$ invariant $(\ell=1, \ldots, N)$. Therefore, $X_{j}$ is reduced by each $\mathscr{H}_{\ell}$. We denote the reduced part of $X_{j}$ to $\mathscr{H}_{\ell}$ by $X_{j}^{(\ell)}$. Then, we have by (23)

$$
\begin{aligned}
U_{\ell} e^{i t X_{j}^{(\ell)}} U_{\ell}^{-1} & =e^{i t^{2} \sum_{k<m}^{2 n} J_{k m}\left(T^{-1}\right)_{k j}\left(T^{-1}\right)_{m j} / 2} e^{i t q_{1}} \cdots e^{i t q_{n}} e^{i t p_{1}} \cdots e^{i t p_{n}} \\
& =e^{i t \bar{X}_{j}(\mathbf{q}, \mathbf{p} ; T)} .
\end{aligned}
$$

Thus, (22) follows.

Theorem 19 tells us that every Weyl representation of the $\Theta-$ GCCR on a separable Hilbert space is unitarily equivalent to a direct sum of the $T$-Schrödinger representation of the $\Theta$ GCCR, where $T \in M_{\Theta}$ is arbitrary.

The next corollary immediately follows from Theorem 19.

Corollary 20. Let $\left\{X_{j}\right\}_{j=1}^{2 n}$ be an irreducible Weyl representation of the $\Theta-G C C R$ on a separable Hilbert space $\mathscr{H}$. Then, for each $T \in M_{\Theta}$, there exists a unitary operator $U: \mathscr{H} \rightarrow$ $L^{2}\left(\mathbb{R}^{n}\right)$ such that

$$
U X_{j} U^{-1}=\bar{X}_{j}(\mathbf{q}, \mathbf{p} ; T), \quad j=1, \ldots, 2 n .
$$

The following result shows that the arbitrariness of the choice of $T$ in the $T$-Schrödinger representation of the $\Theta$ GCCR is implemented by unitary operators.

Corollary 21. Let $S, T \in M_{\Theta}$. Then, there exists a unitary operator $V$ on $L^{2}\left(\mathbb{R}^{n}\right)$ such that

$$
V \bar{X}_{j}(\mathbf{q}, \mathbf{p} ; S) V^{-1}=\bar{X}_{j}(\mathbf{q}, \mathbf{p} ; T), \quad j=1, \ldots, 2 n .
$$

Proof. We need only to apply Corollary 20 to the case where $X_{j}=\bar{X}_{j}(\mathbf{q}, \mathbf{p} ; S)$.

Remark 22. As in the case of non-Weyl representations of CCR, for non-Weyl representations of the $\Theta-G C C R$, the conclusion of Theorem 19 does not hold in general. Examples of such representations of the $\Theta-$ GCCR can be constructed from non-Weyl representations of CCR (e.g., [15, 16, 20, 21]). A detailed description of some examples is given in [5].

\section{Appendix}

\section{Some Properties of Self-Adjoint Operators Satisfying Relations of Weyl Type}

Let $N \geq 2$ be an integer, and let $A_{j}(j=1, \ldots, N)$ be selfadjoint operators on a Hilbert space $\mathscr{H}$ satisfying relations of Weyl type:

$$
e^{i t A_{j}} e^{i s A_{k}}=e^{-i t s a_{j k}} e^{i s A_{k}} e^{i t A_{j}}, t, s \in \mathbb{R}, j, k=1, \ldots, N,
$$

where $a_{j k}$ 's are real constants. It follows that $a_{j k}$ is antisymmetric in $(j, k)$ :

$$
a_{j k}=-a_{k j}, \quad j, k=1, \ldots, N .
$$

The unitarity of $e^{i t A_{j}}$ and functional calculus imply that

$$
\exp \left(i s e^{i t A_{j}} A_{k} e^{-i t A_{j}}\right)=\exp \left(i s\left(A_{k}-t a_{j k}\right)\right), \quad s, t \in \mathbb{R} \text {. }
$$

Hence, we have the operator equality

$$
e^{i t A_{j}} A_{k} e^{-i t A_{j}}=A_{k}-t a_{j k}, \quad t \in \mathbb{R}, j, k=1, \ldots, N .
$$

For a linear operator $A$ on a Hilbert space, we denote the spectrum of $A$ by $\sigma(A)$.

Proposition A.1. Suppose that there exists a pair $(j, k)$ such that $a_{j k} \neq 0$ (hence, $j \neq k$ ). Then,

$$
\sigma\left(A_{j}\right)=\mathbb{R}, \quad \sigma\left(A_{k}\right)=\mathbb{R} .
$$

Moreover, $A_{j}$ and $A_{k}$ are purely absolutely continuous.

Proof. By (A.4) and the unitary invariance of spectrum, we have $\sigma\left(A_{k}\right)=\sigma\left(A_{k}-t a_{j k}\right)$ for all $t \in \mathbb{R}$. Since $a_{j k} \neq 0$, this implies the second equation of (A.5). By (A.2), we have $a_{k j} \neq 0$. Hence, by considering the case of $(j, k)$ replaced by $(k, j)$, we obtain the first equation of (A.5).

Relation (A.4) means that $\left(A_{k}, A_{j}\right)$ is a weak Weyl representation of the CCR with one degree of freedom [14, 15, 24]. Hence $A_{j}$ is purely absolutely continuous $[14,15]$. Similarly, we can show that $A_{k}$ is purely absolutely continuous.

Proposition A.2. Let $j$ and $k$ be fixed. Then, for all $\psi \in$ $D\left(A_{j}\right) \cap D\left(A_{j} A_{k}\right), \psi$ is in $D\left(A_{k} A_{j}\right)$ and

$$
\left[A_{j}, A_{k}\right] \psi=i a_{j k} \psi
$$

Proof. An easy exercise (use (A.4)).

For each function $f \in C_{0}^{\infty}\left(\mathbb{R}^{N}\right)$ and each vector $\psi \in \mathscr{H}$, we define a vector $\psi_{f}$ by

$$
\psi_{f}:=\int_{\mathbb{R}^{N}} f(\mathbf{t}) e^{i t_{1} A_{1}} \cdots e^{i t_{N} A_{N}} \psi d \mathbf{t}
$$


where $\mathbf{t}=\left(t_{1}, \ldots, t_{N}\right) \in \mathbb{R}^{N}$ and the integral on the righthand side is taken in the strong sense. We introduce

$$
\mathscr{D}_{0}:=\operatorname{Span}\left\{\psi_{f} \mid \psi \in \mathscr{H}, f \in C_{0}^{\infty}\left(\mathbb{R}^{N}\right)\right\} \text {, }
$$

where $\operatorname{Span}\{\cdots\}$ denotes the subspace algebraically spanned by the vectors in the set $\{\cdots\}$. It is easy to see that $\mathscr{D}_{0}$ is dense in $\mathscr{H}$.

For $f: \mathbb{R}^{N} \rightarrow \mathbb{C}$ (the set of complex numbers), we set $\|f\|_{1}:=\int_{\mathbb{R}^{N}}|f(\mathbf{t})| d \mathbf{t}$.

Lemma A.3. Let $f_{n}, f \in C_{0}^{\infty}\left(\mathbb{R}^{N}\right)$ such that $\left\|f_{n}-f\right\|_{1} \rightarrow$ $0(n \rightarrow \infty)$. Then, $\left\|\psi_{f_{n}}-\psi_{f}\right\| \rightarrow 0(n \rightarrow \infty)$.

Proof. Since $e^{i t_{j} A_{j}}$ is unitary, we have $\left\|\psi_{f_{n}}-\psi_{f}\right\| \leq \| f_{n}-$ $f\left\|_{1}\right\| \psi \|$. Thus, the desired result follows.

For each $j=1, \ldots, N$, we define a function $g_{j}$ on $\mathbb{R}^{N}$ by

$$
g_{j}(\mathbf{t}):=\left\{\begin{array}{ll}
0 & \text { for } j=1 \\
\sum_{k=1}^{j-1} a_{j k} t_{k} & \text { for } 2 \leq j \leq N,
\end{array} \quad \mathbf{t} \in \mathbb{R}^{N} .\right.
$$

Proposition A.4. (i) For all $t \in \mathbb{R}$ and $j=1, \ldots, N$, $e^{i t A_{j}}$ leaves $\mathscr{D}_{0}$ invariant.

(ii) For each $j=1, \ldots, N, A_{j}$ leaves $\mathscr{D}_{0}$ invariant (i.e., $\left.A_{j} \mathscr{D}_{0} \subset \mathscr{D}_{0}\right)$ and for all $\ell \in \mathbb{N}$,

$$
A_{j}^{\ell} \psi_{f}=(-i)^{\ell} \psi_{F_{j}^{\ell}(f)}, \quad f \in C_{0}^{\infty}\left(\mathbb{R}^{N}\right),
$$

where $F_{j}: C_{0}^{\infty}\left(\mathbb{R}^{N}\right) \rightarrow C_{0}^{\infty}\left(\mathbb{R}^{N}\right)$ is defined by

$$
F_{j}(f):=-\partial_{j} f-i g_{j} f, \quad f \in C_{0}^{\infty}\left(\mathbb{R}^{N}\right),
$$

and $F_{j}^{\ell}$ is the $\ell$ times composition of $F_{j}$ with $F_{j}^{0}:=I$ (identity).

(iii) For all $\ell_{1}, \ldots, \ell_{N} \in \mathbb{N} \cup\{0\}$,

$$
\begin{aligned}
A_{1}^{\ell_{1}} A_{2}^{\ell_{2}} \cdots A_{N}^{\ell_{N}} \psi_{f} & \\
& =(-i)^{\ell_{1}+\cdots+\ell_{N}} \psi_{F_{1}^{\ell_{1}} \cdots F_{N}^{\ell_{N}}(f)}, \quad f \in C_{0}^{\infty}\left(\mathbb{R}^{N}\right) .
\end{aligned}
$$

Proof. (i) Let $\psi_{f}$ be as above. Then, we have $e^{i t A_{j}} \psi_{f}=$ $\int_{\mathbb{R}^{N}} f(\mathbf{t}) e^{i t A_{j}} e^{i t_{1} A_{1}} \cdots e^{i t_{N} A_{N}} \psi d \mathbf{t}$. By (A.1), we have

$$
\begin{aligned}
& e^{i t A_{j}} e^{i t_{1} A_{1}} \cdots e^{i t_{N} A_{N}} \\
& \quad=e^{-i t g_{j}(\mathbf{t})} e^{i t_{1} A_{1}} \cdots e^{i t_{j-1} A_{j-1}} e^{i\left(t_{j}+t\right) A_{j}} e^{i t_{j+1} A_{j+1}} \cdots e^{i t_{N} A_{N}} .
\end{aligned}
$$

Hence,

$$
e^{i t A_{j}} \psi_{f}=\psi_{f_{j}^{(t)}}
$$

with

$$
f_{j}^{(t)}(\mathbf{t}):=f\left(t_{1}, \ldots, t_{j-1}, t_{j}-t, t_{j+1}, \ldots, t_{N}\right) e^{-i t g_{j}(\mathbf{t})} .
$$

It is easy to see that $f_{j}^{(t)}$ is in $C_{0}^{\infty}\left(\mathbb{R}^{N}\right)$. Hence, $\psi_{f_{j}^{(t)}} \in \mathscr{D}_{0}$. Thus, $e^{i t A_{j}}$ leaves $\mathscr{D}_{0}$ invariant.

(ii) By (A.14), we have for all $t \in \mathbb{R} \backslash\{0\},\left(e^{i t A_{j}}-1\right) \psi_{f} / t=$ $\psi_{\left(f_{j}^{(t)}-f\right) / t}$. It is easy to see that $\left\|\left(f_{j}^{(t)}-f\right) / t-F_{j}(f)\right\|_{1} \rightarrow$ $0(t \rightarrow 0)$. Hence, by Lemma A.3,

$$
\lim _{t \rightarrow 0} \frac{\left(e^{i t A_{j}}-1\right) \psi_{f}}{t}=\psi_{F_{j}(f)} .
$$

Therefore, $\psi_{f}$ is in $D\left(A_{j}\right)$ and $i A_{j} \psi_{f}=\psi_{F_{j}(f)}$. Hence, (A.10) with $\ell=1$ holds. Then, one can prove (A.10) by induction.

(iii) This easily follows from (ii). result.

Propositions A.2 and A.4 immediately yield the following

Corollary A.5. For all $j, k=1, \ldots, N,\left[A_{j}, A_{k}\right]=i a_{j k}$ on $\mathscr{D}_{0}$.

Theorem A.6. For all $c_{j} \in \mathbb{R}, j=1, \ldots, N, \sum_{j=1}^{N} c_{j} A_{j}$ is essentially self-adjoint on $\mathscr{D}_{0}$ and

$$
e^{i t \overline{\sum_{j=1}^{N} c_{j} A_{j}}}=e^{i t^{2} \sum_{j<k}^{N} a_{j k} c_{j} c_{k} / 2} e^{i t c_{1} A_{1}} e^{i t c_{2} A_{2}} \cdots e^{i t c_{N} A_{N}},
$$

where for a closable operator $C, \bar{C}$ denotes the closure of $C$.

Proof. For each $t \in \mathbb{R}$, we define an operator $U(t)$ by

$$
U(t):=e^{i t^{2} \sum_{j<k}^{N} a_{j k} c_{j} c_{k} / 2} e^{i t c_{1} A_{1}} e^{i t c_{2} A_{2}} \cdots e^{i t c_{N} A_{N}} .
$$

By using (A.1), one can show that $\{U(t)\}_{t \in \mathbb{R}}$ is a strongly continuous one-parameter unitary group. Hence, by the Stone theorem, there exists a unique self-adjoint operator $A$ on $\mathscr{H}$ such that $U(t)=e^{i t A}, t \in \mathbb{R}$. By Proposition A.4, $U(t)$ leaves $\mathscr{D}_{0}$ invariant and strongly differentiable on $\mathscr{D}_{0}$ with

$$
\left.\frac{d U(t) \psi}{d t}\right|_{t=0}=i \sum_{j=1}^{N} c_{j} A_{j} \psi, \psi \in \mathscr{D}_{0} .
$$

Hence, $\mathscr{D}_{0}$ is a core of $A$ (e.g., [23, Theorem VIII.10]). Hence $A \psi=\sum_{j=1}^{N} c_{j} A_{j} \psi, \psi \in \mathscr{D}_{0}$. Thus, the desired result follows.

For all $c_{j} \in \mathbb{R}, j=1, \ldots, N$, we set

$$
A(\mathbf{c}):=\overline{\sum_{j=1}^{N} c_{j} A_{j}}, \quad \mathbf{c}=\left(c_{1}, \ldots, c_{N}\right) \in \mathbb{R}^{N} .
$$

Corollary A.7. For all $\mathbf{c}, \mathbf{d} \in \mathbb{R}^{N}$ and $t, s \in \mathbb{R}$,

$$
e^{i t A(\mathbf{c})} e^{i s A(\mathbf{d})}=e^{-i t s \sum_{j, k=1}^{N} a_{j k} c_{j} d_{k}} e^{i s A(\mathbf{d})} e^{i t A(\mathbf{c})} .
$$

Proof. By direct computations using (A.17) and (A.1).

\section{Acknowledgment}

This work is supported by the Grant-In-Aid no. 24540154 for Scientific Research from Japan Society for the Promotion of Science (JSPS). 


\section{References}

[1] S. Doplicher, K. Fredenhargen, and J. Roberts, "The quantum structure of spacetime at the Planck scale and quantum fields," Communications in Mathematical Physics, vol. 172, no. 1, pp. 187-220, 1995.

[2] V. Gatral, J. M. Gracia-Bondia, and F. Ruiz Ruiz, "Positiondependent noncommutative products: classical construction and field theory," Nuclear Physics B, vol. 727, no. 3, pp. 513-536, 2005.

[3] Y.-G. Miao, H. J. W. Muller-Kirsten, and D. K. Park, "Chiral bosons in noncommutative spacetime," Journal of High Energy Physics, vol. 08, p. 038, 2003.

[4] Y. Habara, "A new approach to scalar field theory on noncommutative space," Progress of Theoretical Physics, vol. 107, no. 1, pp. 211-230, 2002.

[5] A. Arai, "Representations of a quantum phase space with general degrees of freedom," Mathematical Physics Preprint Archive 09-122. In press.

[6] A. Arai, "Hilbert space representations of quantum phase spaces with general degrees of freedom," RIMS Kôkyûroku, vol. 1705, pp. 51-62, 2010.

[7] L. Gouba and F. G. Scholtz, "On the uniqueness of unitary representations of the non-commutative Heisenberg-Weyl algebra," Canadian Journal of Physics, vol. 87, no. 9, pp. 995-997, 2009.

[8] L. Jonke and S. Meljanac, "Representations of non-commutative quantum mechanics and symmetries," European Physical Journal C, vol. 29, pp. 433-439, 2003.

[9] K. Li and J. Wang, "The topological AC effect on noncommutative phase space," European Physical Journal C, vol. 50, no. 4, pp. 1007-1011, 2007.

[10] L. R. Riberio, E. Passos, C. Furtado, and J. R. Nascimento, "Landau analog levels for dipoles in non-commutative space and phase space," European Physical Journal C, vol. 56, no. 4, pp. 597-606, 2008.

[11] J.-Z. Zhang, "Consistent deformed bosonic algebra in noncommutative quantum mechanics," International Journal of Modern Physics A, vol. 23, no. 09, pp. 1393-1403, 2008.

[12] K. Schmüdgen, Unbounded Operator Algebras and Representation Theory, Birkhäuser, Basel, Switzerland, 1990.

[13] C. R. Putnam, Commutation Properties of Hilbert Space Operators and Related Topics, Springer, Berlin, Germany, 1967.

[14] A. Arai, "Generalized weak Weyl relation and decay of quantum dynamics," Reviews in Mathematical Physics, vol. 17, no. 9, pp. 1071-1109, 2005.

[15] K. Schmüdgen, "On the Heisenberg commutation relation. I," Journal of Functional Analysis, vol. 50, no. 1, pp. 8-49, 1983.

[16] K. Schmüdgen, "On the Heisenberg commutation relation. II," Journal of Functional Analysis, vol. 19, no. 1, pp. 601-671, 1983.

[17] H. P. Robertson, "The uncertainty principle," Physical Review, vol. 34 , no. 1, pp. 163-164, 1929.

[18] I. Satake, Linear Algebra, Shokabo, Tokyo, Japan, 1974.

[19] M. Reed and B. Simon, Methods of Modern Mathematical Physics II: Fourier Analysis, Self-Adjointness, Academic Press, New York, NY, USA, 1975.

[20] A. Arai, "Representation-theoretic aspects of two-dimensional quantum systems in singular vector potentials: canonical commutation relations, quantum algebras, and reduction to lattice quantum systems," Journal of Mathematical Physics, vol. 39, no. 5, pp. 2476-2498, 1998.
[21] B. Fuglede, "On the relation $P Q-Q P=-i I$," Mathematica Scandinavica, vol. 20, pp. 79-88, 1967.

[22] J. von Neumann, "Die Eindeutigkeit der Schrödingerschen operatoren," Mathematische Annalen, vol. 104, no. 1, pp. 570-578, 1931.

[23] M. Reed and B. Simon, Methods of Modern Mathematical Physics I: Functional Analysis, Academic Press, New York, NY, USA, 1972.

[24] A. Arai and Y. Matsuzawa, "Construction of a Weyl representation from a weak Weyl representation of the canonical commutation relation," Letters in Mathematical Physics, vol. 83, no. 2, pp. 201-211, 2008. 


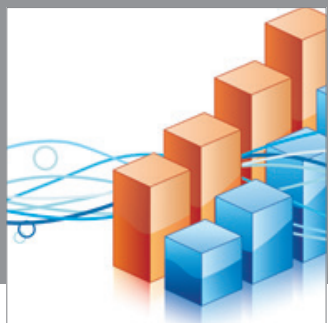

Advances in

Operations Research

mansans

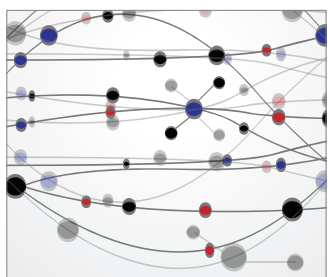

The Scientific World Journal
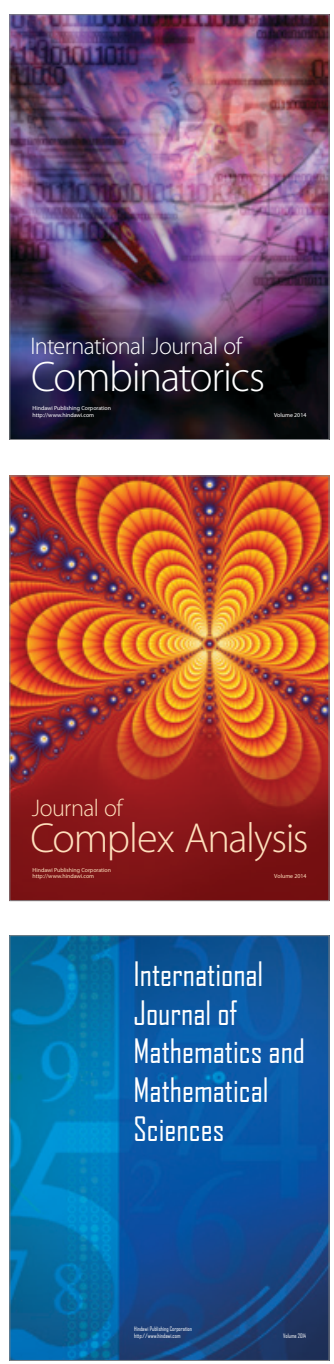
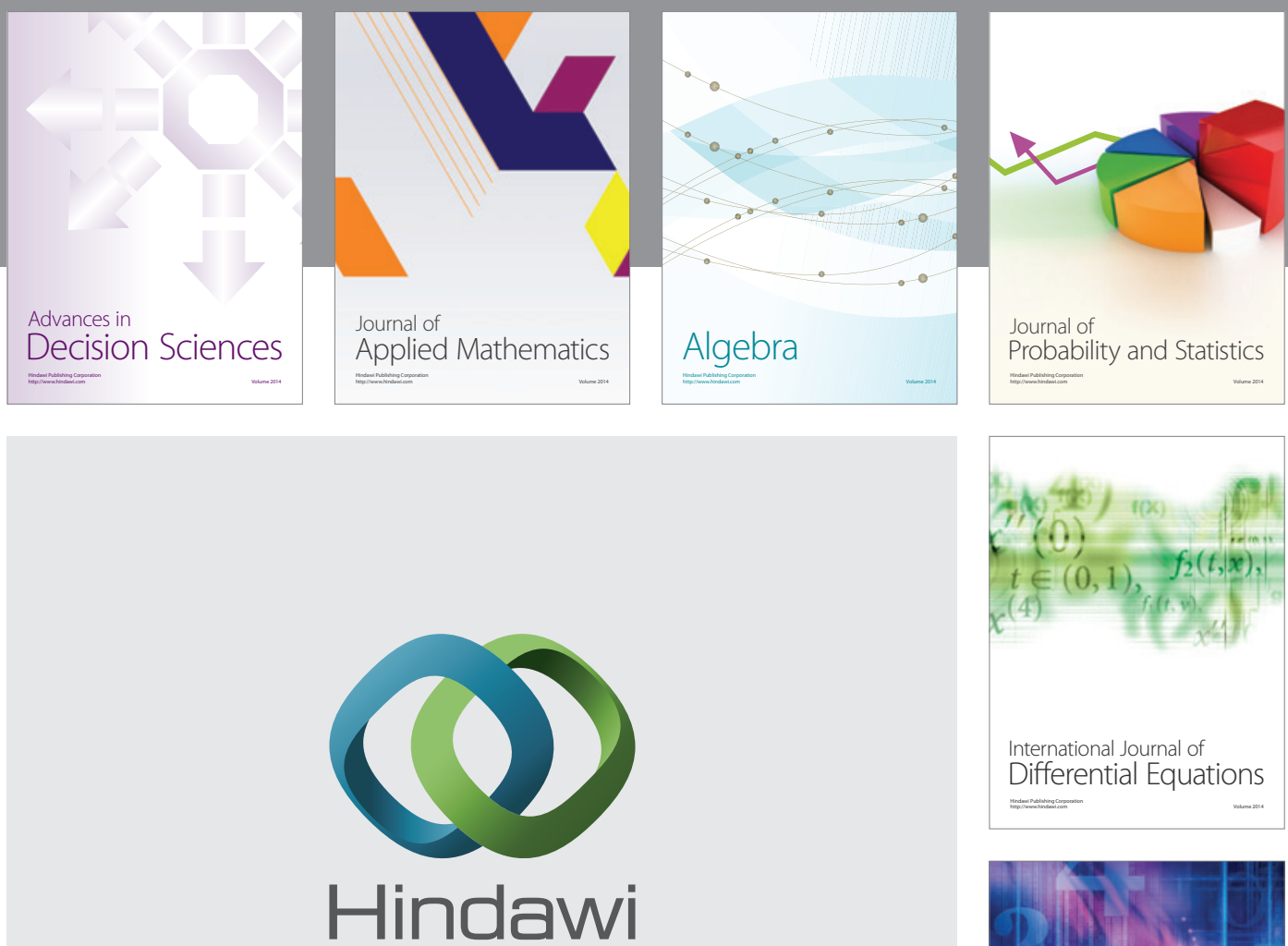

Submit your manuscripts at http://www.hindawi.com
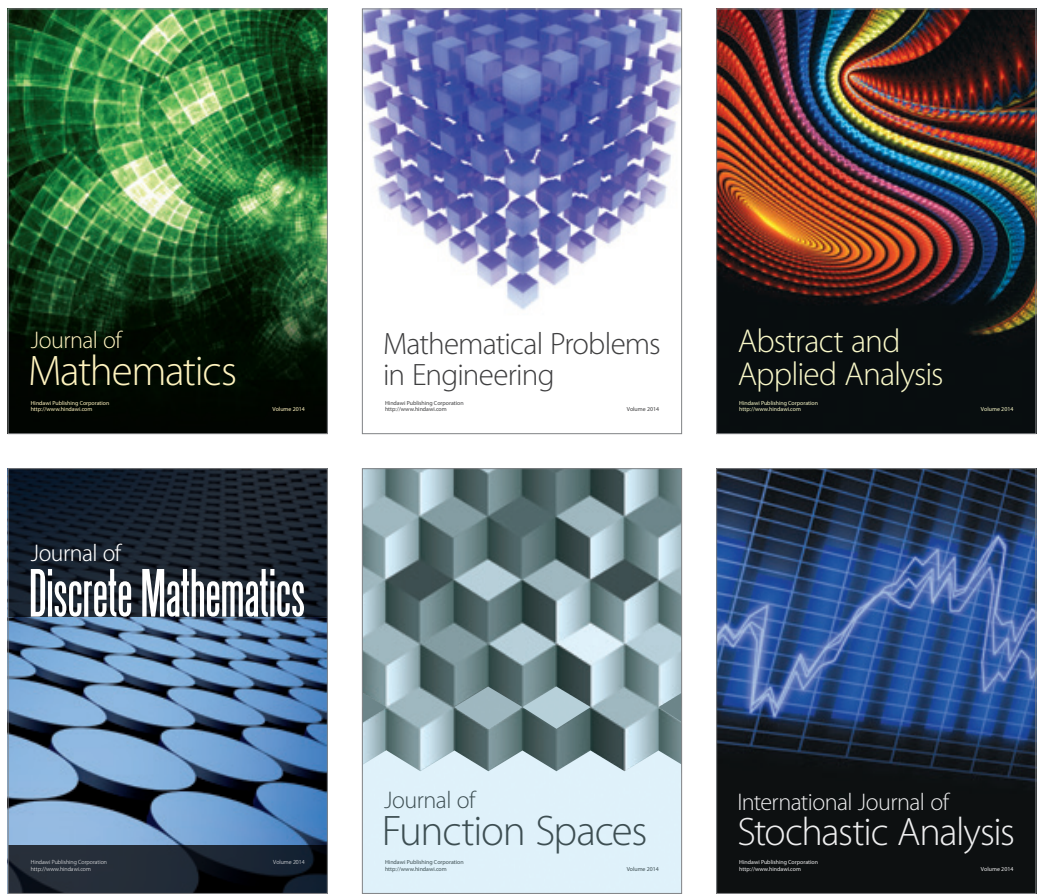

Journal of

Function Spaces

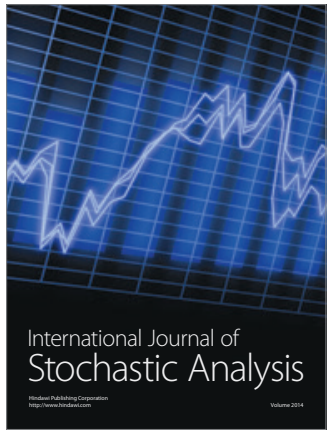

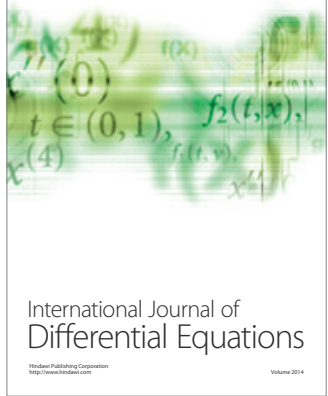
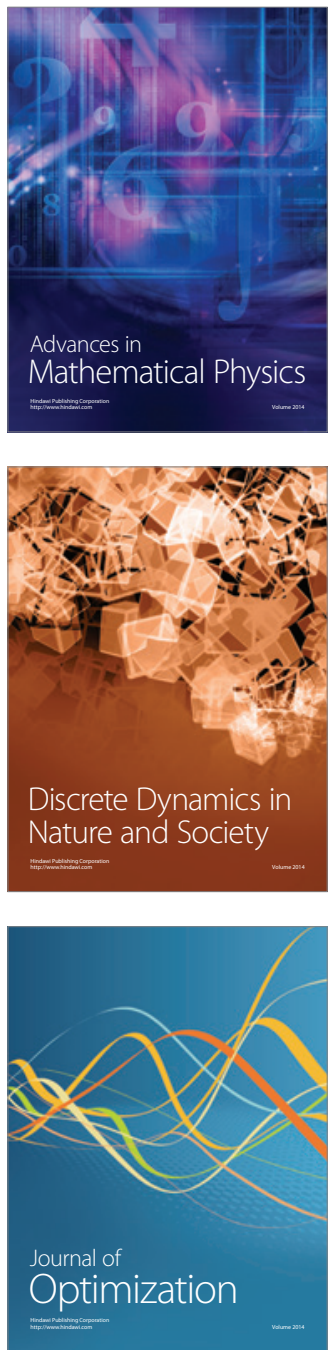